\title{
MAESTROS PARA LA DEMOCRACIA. LA FORMACIÓN INICIAL DEL MAGISTERIO EN ESPAÑA CON LA LEY DE ORDENACIÓN GENERAL DEL SISTEMA EDUCATIVO (1990)
}

\author{
DOI: http://dx.doi.org/10.1590/2236-3459/70869
}

\author{
Teresa González Pérez \\ Universidad de La Laguna, San Cristóbal de La Laguna/Santa Cruz de Tenerife, España
}

$\cos 80$

\begin{abstract}
Resumen
En España, en el marco de la sociedad democrática, después de cuatro décadas de dictadura, se gestó un nuevo modelo de formación del magisterio. Nuestro objetivo consiste en estudiar el modelo de formación inicial del magisterio con la Ley de Ordenación General del Sistema Educativo (1990) y el perfil profesional emanado de su diseño. Realizamos el análisis del discurso de la reforma, de la legislación y programas de estudio así como una revisión bibliográfica. El estudio nos permite conocer la política educativa para la formación del magisterio en los inicios de la democracia española. La reforma educativa generó transformaciones en las instituciones, en los programas y prácticas pedagógicas, pero no mejoró el nivel académico de los egresados. Con estas aportaciones contribuimos a la reflexión sobre los programas de formación docente.

Palabras clave: democracia, reformas, legislación educativa, formación inicial del magisterio, planes de estudio.
\end{abstract}

\section{PROFESSORES PARA A DEMOCRACIA. A FORMAÇÃO INICIAL DE PROFESSORES EM ESPANHA COM A LEI SOBRE O SISTEMA GERAL DE EDUCAÇÃO (1990)}

\begin{abstract}
Resumo
Na Espanha, no âmbito da sociedade democrática, depois de quatro décadas de ditadura, foi concebido um novo modelo de formação dos professores. Nosso objetivo é estudar o modelo de formação inicial de professores com a Lei sobre o Sistema Geral de Educação (1990) e o perfil profissional que emanava de sua concepção. Analisamos o discurso da reforma, a legislação, os programas de estudo e realizamos uma revisão da literatura. $O$ estudo nos permite conhecer a política educacional para a formação de professores no início da democracia espanhola. A reforma da educação gerou transformações nas instituições, programas e práticas de ensino, mas não melhorou o nível académico dos licenciados. Com estes aportes buscamos contribuir para a reflexão sobre os programas de formação de professores.

Palavras-chave: democracia, reforma, legislação da educação, formação inicial de professores, currículo.
\end{abstract}

\section{TEACHERS FOR DEMOCRACY. THE INITIAL TRAINING OF TEACHERS IN THE LAW ON GENERAL EDUCATION SYSTEM (1990)}

\section{Abstract}

In Spain, within the framework of democratic society, after four decades of dictatorship, a new model of teacher education was developed. Our objective is to study the model of initial teacher training with the Law of General 
Management of the Educational System (1990) and the professional profile emanated from its design. We analyze the discourse of reform, legislation and programs of study as well as a bibliographic review. The study allows us to know the educational policy for the training of teachers in the beginnings of Spanish democracy. The educational reform generated changes in the institutions, in the programs and pedagogical practices, but did not improve the academic level of the graduates. With these contributions we contribute to the reflection on the programs of teacher training.

Keywords: democracy, reforms, educational legislation, initial teacher training, curricula.

\section{LES ENSEIGNANTS POUR LA DÉMOCRATIE. LA FORMATION INITIALE DES ENSEIGNANTS EN ESPAGNE AVEC LA LOI SUR LE SYSTĖME D'ÉDUCATION GÉNÉRALE (1990)}

\section{Résumé}

En Espagne, dans le cadre d'une société démocratique, après quatre décennies de dictature, un nouveau modèle de formation des enseignants a été conçu. Notre objectif est d'étudier le modèle de la formation initiale des enseignants à la Loi sur le système de l'enseignement général (1990) et profil professionnel émané de sa conception. Nous analysons le discours de la réforme, la législation et les programmes d'études et d'une revue de la littérature. L'étude nous permet de connaître la politique éducative pour la formation des enseignants au début de la démocratie espagnole. La réforme de l'éducation a généré des transformations dans les institutions, les programmes et les pratiques d'enseignement, mais n'a pas amélioré le niveau académique des diplômés. Grâce à ces contributions, nous contribuons à la réflexion sur les programmes de formation des enseignants.

Mots-clés: démocratie, réforme, législation sur l'éducation, formation initiale des enseignants, programmes. 


\section{Introducción}

- n España desde la etapa de la transición democrática la educación también se fue transformando, producto de una mezcla de viejas y nuevas ideas con cambios metodológicos. En un periodo de cambios legislativos y de efervescencia social y cultural, la normativa experimentó notables modificaciones. Las políticas educativas generaron cambios, cuyo grado de intensidad depende de los distintos gobiernos y de las ideologías dominantes, que contribuyen a la transformación de la sociedade. (TORRES, 2011, p. 171). En ninguna época el campo de la educación ha subsistido a los intentos de modificación, a reformas de todo tipo, ni a la persistente crítica desde distintos sectores. (BOLÍVAR, 2006).

La legitimación de la escuela democrática y plural, como garante de la igualdad de oportunidades culturales para todos y todas, fue un proceso paulatino que concluyó con el siglo XX, superando el atraso de décadas anteriores y convirtiendo a España en un país más europeo. Para ello afrontó la mejora de la formación inicial. Porque modernizar la educación implicaba necesariamente modernizar al profesorado y sus estructuras formativas. La formación inicial de maestros y maestras ha sido producto de la evolución política. En el caso español la evolución fue lenta y la transformación requirió de un esfuerzo permanente de los gobiernos democráticos, para mejorar la herencia pedagógica. (MOLERO, 2000, p. 75).

En las últimas décadas, el magisterio ha sufrido transformaciones importantes, tanto en sus características y contenidos como en su proyección social y cultural. El discurso formativo ha estado marcado por una serie de orientaciones, de acuerdo con unas determinadas perspectivas educativas. El sistema educativo siempre ha situado la formación docente en el contexto de un discurso ambivalente. Por un lado, la vieja retórica de la importancia de esta formación y, por otro, la precaria situación académica y escasa consideración social. Históricamente, se ha considerado una profesión de segundo orden. Sin embargo, se requería una serie de conocimientos objetivos prefijados y un conocimiento profesional, o la capacidad de generar conocimiento pedagógico. Poseer saberes formales equivalía a asumir la capacidad de enseñarlos, y también tomar decisiones sobre la práctica educativa.

En 1987 el Ministerio de Educación y Ciencia (MEC) presentó el proyecto de Reforma del Sistema Educativo. Se trataba de una propuesta para debatir, que partía del reconocimiento del ritmo acelerado de la sociedad en cuanto a innovaciones. (PROYECTO DE REFORMA, 1987, p. 23). Más tarde, en 1989 se concretó dicha propuesta en el Libro Blanco para la Reforma del Sistema Educativo y fines de la educación (LIBRO BLANCO, 1989, p. 23). La Ley Orgánica de Ordenación del Sistema Educativo (LOGSE, 3 de octubre de 1990) reestructuraba la educación española en base a los avances experimentados en la sociedad, así como equiparaba la educación con los países de su entorno. Entre otras innovaciones se observa la prolongación de la educación obligatoria en dos años, a la vez que eliminaba el desajuste entre el final de la educación obligatoria, situado hasta ese momento en los 14 años, y el comienzo de la edad laboral (16 años). Al mismo tiempo reducía la duración de la escolaridad primaria, que cedió dos años en beneficio de la educación secundaria. Igualmente, se configuró un diseño curricular de carácter abierto, ya que los centros y comunidades educativas disponían de un margen de autonomía más amplio para elaborar su proyecto curricular específico, denominado Proyecto Curricular de Centro. 
Además, la Logse reconocía la discriminación entre sexos, y establecía el principio de igualdad de oportunidades entre sexos. La primera ley de la democracia que adaptaba la normativa al nuevo marco legislativo reconocía, por primera vez, la discriminación educativa en orden al sexo y estableció el principio de no discriminación, fijando la igualdad entre sexos (artículo $\left.2^{\circ}\right)^{1}$.

El panorama del magisterio mejoró, en cierta medida, como consecuencia de la evolución legislativa. Unos cambios educativos prescritos y legislados, para que fueran efectivos tenían que adaptarse a la cultura escolar existente y rediseñarse en los contextos educativos. (BOLÍVAR, 2013, p. 64). La formación inicial del magisterio se vio afectada por la reforma educativa, y desde el propio ministerio se elaboraron propuestas que contribuyeron al debate intelectual. En la década de los ochenta y noventa, se realizaron una serie de Seminarios Estatales sobre la formación de profesorado por diferentes lugares de la geografía española. Los debates en los que participaban representantes de las Escuelas Universitarias recogían el sentir de los sectores implicados, atendían a las preocupaciones y a la urgencia de mejorar la situación del magisterio. En 1987 se creó la Revista Interuniversitaria de Formación del Profesorado como órgano de difusión de este foro, con una línea editorial coherente con la inquietud por la formación inicial del profesorado. La citada Revista, en diferentes editoriales, cuestionaba el perfil profesional. En el primer número de la Revista Interuniversitaria de Formación del Profesorado (1988, p. 5-7) el Consejo Editorial se preguntaba ¿Qué pasa con la formación del profesorado? ¿Qué sucede con la reforma de los centros que hasta el presente se vienen ocupando de tal problemática? Repasaba la serie de reuniones científicas, seminarios de diversa índole, donde manifestaron la preocupación por la formación inicial. Así puntualizaban "El deseo de un cambio en profundidad viene de lejos". José Emilio Palomero Pescador escribió, en la Presentación del oㅡ 2 (1988, p. 10), sobre la reforma curricular en la formación inicial del profesorado. En esta línea incidía en la necesidad de una rigurosa revisión que exija una preparación científica, metodológica y profesional del estudiantado acorde con la sociedad moderna y cambiante ${ }^{2}$.

En la última década del siglo XX, varios expertos coincidían en la importancia de modificar los programas de formación de maestros, y en la necesidad de cambiar la imagen de las escuelas, para lograr una interrelación y proyección de la educación. (IMBERNÓN, 1997; MOLERO, 2000; ZABALZA, 1997; TORRES, 2011).

El objetivo de este trabajo es estudiar el modelo de formación inicial del magisterio con la Ley de Ordenación General del Sistema Educativo (1990) y analizar el perfil profesional emanado de su diseño.

Para la investigación hemos empleado la metodología de análisis históricoeducativa. En primer lugar analizamos la información que nos suministraron distintas fuentes, llevando a cabo un estudio sistemático de la legislación y de la bibliografía. Además

\footnotetext{
${ }^{1}$ Los diseños curriculares recogieron las orientaciones y los objetivos, los contenidos y la metodología para la aplicación de la coeducación. Los diseños curriculares en Educación Infantil, Educación Primaria y Secundaria Obligatoria introducían el principio de igualdad, aspectos básicos que se hicieron públicos a través de una serie de Reales Decretos que fijaron los aspectos básicos del currículo. El 25 de junio de 1991 se publicó en el B.O.E. el calendario de aplicación de la nueva ordenación del sistema educativo, estableciéndose las enseñanzas mínimas para los distintos niveles de la enseñanza obligatoria mediante los Reales Decretos 1.006/1991 y 1.007/1991 de 26 de junio de 1991.

2 Este número de la Revista Interuniversitaria de Formación del Profesorado es una Monografía dedicada al III Seminario Estatal de Escuelas de Magisterio celebrado en Teruel entre el 6 y 9 de abril de 1987.
} 
de la consulta de fuentes documentales, consultamos fuentes bibliográficas y hemerográficas. A partir de la consulta de la documentación, realizamos el estudio de la política educativa, siguiendo el discurso de la reforma de la Ley Orgánica de Ordenación del Sistema Educativo (Logse) y su diagnóstico previo en el Libro Blanco (1989). Posteriormente, analizamos la legislación, en relación con los programas de formación inicial del magisterio y su desarrollo curricular. Así mismo, efectuamos una revisión bibliográfica de las interpretaciones y valoraciones ofrecidas por los expertos.

\title{
Diseñar nuevos perfiles docentes en el marco de la reforma educativa
}

La reforma del sistema educativo exigió la renovación de la figura del profesor. Así lo recogió el Libro Blanco (1989, p. 209-210), en el Capítulo XIII, donde refería que una escuela renovada necesitaba de un profesorado renovado. (LIBRO BLANCO, 1989, p. 213). El perfil de profesor que trazaba se combinaba con un conjunto de elementos de innovación y competencias didácticas. Simultáneamente a la reforma del sistema de enseñanzas no universitarias se produjo el proceso de reforma de los estudios de Magisterio. El Ministro de Educación, José Ma Maravall (Ministro entre 1982-1988, bajo la presidencia de Felipe González con el PSOE), consciente de la importancia y alcance de la reforma educativa, proyectaba la formación inicial de los docentes dada la necesidad de "un nuevo profesor para la reforma educativa". Maravall no dudaba de la preparación universitaria, y de impulsar su especialización en este ámbito. Expresaba

\begin{abstract}
España es uno de los países cuyo sistema de formación del profesorado se halla incardinado dentro de la Universidad. Esa inserción no debe ser revisada; por el contrario ha de ser profundizada, persiguiendo una mayor integración en el caso de la formación de profesores de EGB, y una mayor estabilidad en la formación de profesores de secundaria. La Universidad ha de aceptar con todas las consecuencias que la formación de profesores constituye uno de sus cometidos: $\sin$ duda, uno de los de mayor trascendencia para el sistema educativo [...]. La integración de los profesores en departamentos universitarios, la responsabilidad de estos en la configuración de los currícula, unidas a una selectividad idéntica a la que pueda establecerse para cualquier otro tipo de Facultades, permitirán asegurar en el futuro un profesorado de EGB de mejor calidad, dando verdadero sentido universitario a unos centros que hasta ahora han tenido más bien una adscripción nominal a la universidad. (MARAVALL, 1984, p. 73-74).
\end{abstract}

La propia ley (LOGSE, 1990), indujo a la actualización de la Diplomatura de Magisterio, cuya propuesta fue aprobada por la Comisión Académica del Consejo de Universidades el 28 de septiembre de 1992. Los trabajos dirigidos hacia la reforma de los planes de estudio de las Escuelas de Magisterio, constituyeron una oportunidad única y necesaria para potenciar estas Escuelas. También para mejorar la calidad de la formación inicial del profesorado, así como para atender a la demanda de un nuevo modelo de maestro/a, que respondiera a las tareas y responsabilidades que se le atribuían. Los resultados, sin embargo, no respondieron a las expectativas generadas para la elaboración de los nuevos planes. Por el contrario, la multiplicidad de intereses departamentales encontrados, motivó que la elaboración de los planes de estudio se ajustara más al equilibrio de fuerzas entre los intereses citados que a los objetivos de calidad planteados, para la formación eminentemente profesional de los futuros docentes. En el diseño de planes de estudio primó el corporativismo frente a la reflexión sobre el perfil y competencias profesionales. El saber académico y profesional, la fragmentación por el excesivo número 
de materias, la formación científica y la formación psicopedagógica disociada y superflua, no tenía en cuenta el modelo de escuela para el que se pretendía formar y su función educativa. Cuando realmente, es el modelo de escuela el que determina el perfil de los maestros.

La Logse planteaba el paradigma práctico de un profesor reflexivo y orientado a la investigación. La formación de los maestros y maestras debía proporcionar las herramientas necesarias para la formación reflexiva de su pensamiento, para actuar desde la investigación acción. La preparación del alumnado, aspirante al magisterio, implicaba una cualificación pedagógica y científica en un periodo de tres años. Sin embargo, en este proceso se dedicaron más a atender deficiencias formativas del estudiantado, priorizando su carácter culturalista frente al profesional, relegando las materias pedagógicas y las prácticas de enseñanza. Finalmente se registró una cierta diversidad en la elaboración y desarrollo de los currículos, debido al margen de autonomía disfrutado por las Comisiones de Planes de Estudio de cada distrito universitario. El continuismo del modelo de diplomatura no fue del agrado de los sectores implicados.

De acuerdo con el Real Decreto 1440/1991, "por el que se establece el título universitario oficial de maestro en sus diversas especialidades y las directrices generales de los planes de estudios conducentes a su obtención"3, se concretó en siete especialidades con una asignación determinada de créditos:

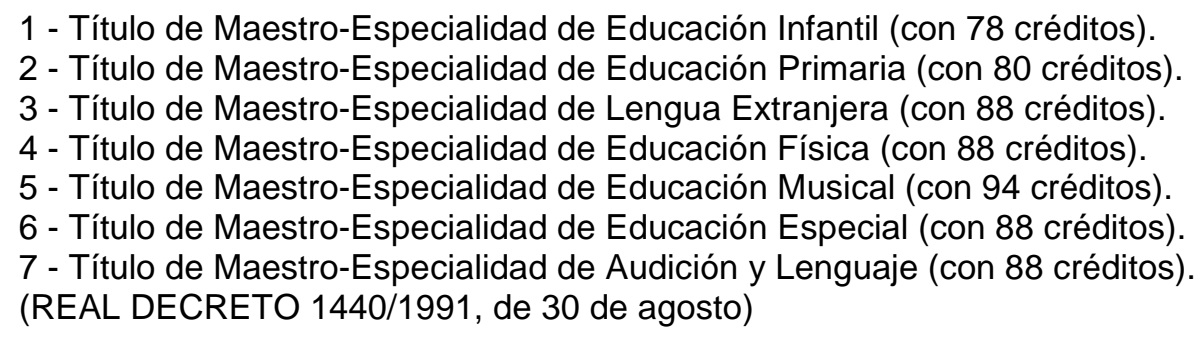

Tal como se desprende de la relación anterior, las especialidades con menor número de créditos eran Educación Infantil y Educación Primaria y la de mayor Educación Musical. Conforme a las Directrices Generales para todo el Estado, los títulos se configuraban con un conjunto de materias troncales (unas materias troncales comunes y otras específicas de cada especialidad) iguales en toda España (ver Anexo); un grupo de materias obligatorias; materias optativas y de libre configuración curricular propias de cada universidad, además del Prácticum. Las materias troncales venían definidas en la matriz de las titulaciones. Pero las materias obligatorias, optativas y libre configuración las determinaba cada Universidad. Un hecho que generó una diversidad de variantes en la formación inicial, según los distritos universitarios. El plan de estudios pretendía homogeneizar unos mínimos contenidos en todos los distritos, que de acuerdo a las directrices generales, elaboran el correspondiente plan de estudios. Si bien, contenían una base común de materias de las ramas pedagógicas, psicológicas y didácticas específicas en todas las especialidades, que proporcionaban la formación teórico-práctica del alumnado, no cubrió las expectativas que suscitaron los nuevos planes de estudio. La sobrecarga del currículum formativo, con un excesivo número de materias por curso (suponían entre 14 y 17 asignaturas), generada por los múltiples intereses del profesorado

${ }^{3}$ Real Decreto 1440/1991, de 30 de agosto. (BOE, 11 de octubre). 
y de los propios departamentos, reveló una serie de deficiencias. Además de la falta de recursos económicos para aplicar la reforma, que condujo al fracaso en el proceso de implantación. La controversia de nuevos planes formativos tropezaba con viejos problemas, que no resolvieron la preparación inicial del colectivo docente.

El desarrollo curricular de la troncalidad, establecida por el Real Decreto 1440/1991, no fue uniforme y experimentó variaciones en su concreción en cada distrito universitario. Pese a que las respectivas Comisiones de Planes de Estudios elaboraron sus propuestas, éstas no fueron llevadas a trámite en su totalidad. En algunas Escuelas Universitarias se implantó el nuevo plan de estudios en sus cinco primeras especialidades. Otras lo hicieron de forma paulatina. $Y$ hubo casos en los que solo se impartieron las especialidades más demandadas en sus contextos. Lo más frecuente fueron las cinco primeras especialidades. Pues en algunas Facultades no disponían de profesorado especializado para hacer frente a las especialidades de Educación Especial y de Audición y Lenguaje.

La estructura del nuevo plan de estudios perseguía un currículo flexible, adaptado a las nuevas exigencias profesionales. Los estudios se articularon en tres cursos divididos en dos semestres cada uno, equivalentes a 15 semanas lectivas de clase. La carga lectiva de la diplomatura, en cada una de sus especialidades, era de un cómputo total de 242 créditos. De los cuales, 188 créditos se corresponden a las materias troncales del Ministerio y obligatorias de Universidad. La suma de 25,5 créditos de libre elección, es decir, materias elegidas libremente por el alumnado entre las impartidas por la Universidad. Y 28,5 créditos en concepto de materias optativas, asignaturas elegidas entre la oferta de 136,5 créditos que se realizaba para cada una de las especialidades del título de Maestro/a. A todo ello añadir los 32 créditos correspondientes al Prácticum (antes denominadas Prácticas de Enseñanza). Los créditos de libre elección se cursaban en segundo y/o tercer curso, después de haber aprobado todas las materias del primer curso. Para poder realizar el Prácticum era requisito haber aprobado un mínimo de 103,5 créditos de materias obligatorias. Consistía en realizar prácticas de docencia, en los distintos niveles de la Educación Primaria, con el objetivo de conocer la estructura de la enseñanza. El Prácticum estaba integrado en la estructura de la carrera. Era fundamental en la formación inicial de maestros y maestras para lograr los objetivos del perfil profesional. En la metodología exigía la intervención activa del profesorado universitario, tanto en su preparación y supervisión como en la reflexión. El Prácticum constituye un componente curricular básico en el nuevo marco del programa formativo, y modificaba la antigua concepción sobre las prácticas de enseñanza. Un nuevo enfoque orientado a enriquecer el aprendizaje teórico académico, que le permitía ampliar conocimientos propios del ejercicio profesional. (ZABALZA, 1998, p. 173). Suponía la inmersión de los estudiantes en centros educativos, con la finalidad de conocerlos directamente, integrar conocimientos teórico-prácticos y adquirir competencias profesionales en todos los ámbitos de actuación. Las prácticas, continuaban siendo supervisadas, por el profesor tutor universitario y el maestro colaborador del centro. En la calificación final también se añadía la valoración de la Memoria que tenía que presentar el alumnado. De esta manera se trataba de interrelacionar la realidad educativa con la formación inicial recibida por el estudiantado, tratando de formar profesionales críticos, reflexivos y comprometidos. El periodo de prácticas requería de una preparación previa del alumnado, con la finalidad de adquirir las competencias educativas específicas de la escuela. Precisaba de un desarrollo curricular provisto de una fundamentación teórica de 
las prácticas a realizar, sus objetivos y metodología. El Prácticum constituye un recurso importante en el proceso global de formación y permite adquirir nuevos conocimientos, desarrollar nuevas habilidades, modificar actitudes, revisar planteamientos, facilitar la integración laboral, etc. La Logse le concedía importancia a la reflexión sobre la práctica, a su investigación-acción. Sin embargo, el modelo era un tanto difuso. Porque los aprendizajes no estaban bien definidos, ni tampoco las expectativas adscritas al perfil profesional, porque estaba escasamente integrado en la carrera. Esa escasa formalización y la desconexión con las otras materias del currículum académico difuminaban sus objetivos. El Prácticum, que ostentaba mayor espacio académico en relación al Plan 1971, debía ser la expresión del modelo de formación pero no era coherente con el modelo formativo ni con las expectativas que se generaban con dichas prácticas.

\section{¿Avance o retroceso en el modelo de formación inicial?}

Las reformas educativas deben propiciar un avance, en sentido amplio. El progreso cualitativo, que necesitaba la educación, se concretaba en la profesionalización docente. Un cúmulo de circunstancias generó recelos y desconfianzas cuando se aprobó la Logse y el modelo de formación inicial del magisterio. Desde algunos sectores vinculados al magisterio, conscientes de que la formación inicial del profesorado constituye la base de la profesionalización, se insistió en que no se llevó a cabo una reforma sino una modificación del plan de estudio de 1971, añadiendo nuevas especialidades, cuando se requería un cambio en la formación inicial del profesorado. (IMBERNÓN, 1997). La reforma recuperó al maestro/a generalista de enseñanza primaria. Incluyó nuevas especialidades consideradas deficitarias como Lengua Extranjera, Educación Musical y Educación Física, junto a otras especialidades consideradas importantes a nivel social, como la Audición y Lenguaje y la Educación Especial. La intención del legislador era significar la profesión y distinguir la formación. Por ello se recuperó la antigua denominación de Maestros, pero desde el ámbito institucional restringido y poco coherente con la dimensión social del magisterio. La Diplomatura de Maestros y Maestras especialistas continuaba adscrita a las Escuelas Universitarias o a Facultades de Educación, según la organización de los distritos universitarios. La carrera de Magisterio no se identificaba con unos estudios relevantes, desde el punto de vista académico y profesional, al exigirse un menor tiempo para cursarlas. Es decir, se transformaron y redujeron las licenciaturas a cuatro cursos, mientras se mantenían las diplomaturas con tres años académicos. La demanda de alargar la formación y equipararla a la de otras titulaciones universitarias y convertirla en licenciatura era un clamor. Desde las instituciones académicas y algunos sectores profesionales, la diplomatura se quedaba corta para atender la formación.

Tanto la comisión como los numerosos organismos y colectivos que presentaron sugerencias al informe elaborado por aquélla, tuvieron escaso éxito a la hora de convencer a las autoridades ministeriales acerca de la necesidad de ampliar al grado de Licenciatura la formación inicial de los maestros, única opción realista para hacer frente a las nuevas y crecientes demandas que la sociedad proyecta sobre profesores y profesoras, "dotándoles de una formación adecuada que les permita desempeñar adecuadamente los múltiples papeles que luego les vamos a exigir que desempeñen. (ESTEVE, 1996, p. 78).

La titulación gestada repetía viejos errores, alejando a los maestros del modelo 
profesional. Se recuperó la denominación del título de Maestro, pero no la del Centro académico que ostentaría una nueva configuración administrativa. Más bien creaba una diversidad de modelos de maestro/maestra generalista/especialista, en una amalgama de centros universitarios. El proyecto de reforma de la formación del profesorado, realizado en 1984, por un equipo de expertos a instancias del MEC, propuso la integración de las Escuelas y estudios de Ciencias de la Educación en un departamento universitario. La Logse, sin definir previamente el modelo de instituciones para la formación de profesorado, autorizó la adscripción a centros de diferente naturaleza. De manera que la legislación permitió la transformación de las Escuelas Universitarias de Formación del Profesorado en Centros Superiores de Educación o de Formación del Profesorado. En otros casos, la formación inicial del magisterio se adscribió a Facultades de Educación, a Facultades de Humanidades, Facultades de Ciencias Sociales, Centros de Formación del Profesorado, a Escuelas de Educación, donde no existía Facultad de Educación o no había antecedentes de estudios de Pedagogía. La Ley Orgánica de Ordenación General del Sistema Educativo (1990) contemplaba la posibilidad de crear Centros Superiores de Formación del Profesorado o Centros Superiores de Educación ${ }^{4}$. Algunas Escuelas Universitarias fueron absorbidas por las Facultades de Ciencias de la Educación existentes. No obstante, el mapa institucional fue muy variado, abarcando Facultades de Humanidades donde se incluye Educación, Facultades de Ciencias Sociales y Educación, o bien Escuelas de Educación. De manera que la situación académica de la formación del magisterio continuaba siendo un tanto marginal dentro de la universidad. Dado ese carácter precario que le confería la titulación de ciclo corto. Tanto las funciones como las competencias quedaban sin articular. De manera que las Escuelas Universitarias tuvieron que hacer su propia transición, y adaptarse a los cambios según las circunstancias de cada una.

La formación inicial del magisterio ha ido evolucionando al ritmo de las políticas educativas. La política educativa de la democracia supuso una ruptura curricular y metodológica con la etapa anterior. De alguna forma, se puede interpretar que las prácticas docentes son inmunes a los cambios políticos. Dependen de la mentalidad y de las dinámicas profesionales de los maestros y maestras. Según Pérez Gómez (1993, p. 46) se trataba de la "reconstrucción del pensamiento pedagógico de los futuros docentes". La reforma curricular de la formación inicial había sido aclamada desde los distintos sectores implicados. Una preparación científica, metodológica y profesional del estudiantado, acorde con la sociedad cambiante y moderna, tenía que afrontar una rigurosa revisión curricular. El modelo de formación del magisterio era un modelo semiprofesional e insuficiente a nivel académico. La Logse dejó en evidencia la formación de maestros, la necesidad de reconocer su función social y revalorizar su papel. (BOLÍVAR, 2006; COROMINAS, TESOURO; TEIXIDÓ, 2006).

\section{Un modelo regresivo o la pervivencia de la diplomatura universitaria}

El consejo de redacción de la Revista Interuniversitaria de Formación del

\footnotetext{
${ }^{4}$ Logse. Disposición adicional (12.3). La Editorial de la Revista Interuniversitaria de Formación del Profesorado (1993) publicó al respecto en su Editorial "extraña disposición adicional que permite la transformación de las actuales Escuelas en Centros Superiores de Formación del Profesorado, sin antes haber definido la naturaleza de esos hipotéticos y fantasmales centros, como asimismo sus funciones y ámbitos de competencias en los distintos niveles y tipos de formación permanente del profesorado".
} 
Profesorado (1993) escribió: "la reforma de la formación inicial del profesorado y del resto de profesionales relacionados con el mundo educativo se ha llevado a cabo sin acometer con valentía las reformas estructurales necesarias" con el consiguiente perjuicio para las nuevas generaciones. La reforma educativa no resolvió la problemática inicial del colectivo, que sería responsable de la educación de la infancia española. De ahí surgieron algunos interrogantes institucionales y curriculares:

- ¿Dónde se debe realizar la formación inicial del profesorado?

- ¿Cuál es el programa de formación inicial propuesto?

No se aplicó la reforma necesaria por falta de recursos e inversión. De este hecho se deriva la baja calidad de la formación inicial, y no implantar las nuevas especialidades, por no poder contratar profesionales para impartirlas. También las carencias materiales, de infraestructuras y de recursos didácticos entorpecieron la formación. La preocupación de los equipos directivos y del personal docente, de las Escuelas universitarias, se reflejó en la serie de sesiones de trabajo y seminarios que se realizaron, con alta participación de los distintos distritos universitarios.

En la última década del siglo XX varios expertos coincidían en la importancia de modificar la formación de maestros y cambiar la imagen de las escuelas, para lograr una interrelación y proyección de la educación. Para ello había que potenciar unas nuevas relaciones sociedad-escuela, y un nuevo modelo de profesor. (RODRÍGUEZ LESTEGÁS, 1998, p. 2-3). La Unesco definió la formación docente adecuada a su función educadora: "un proceso a la vez abierto hacia las necesidades reales de la sociedad (a diversos niveles: nacional, provincial y local) y pluridisciplinario, es decir, un enfoque "por problema" y un enfoque "participativo" (basado en una actitud de investigación participativa y de investigación-acción)". (UNESCO, 1994, p. 119). Se anticipaba al modelo basado en competencias ${ }^{5}$.

La Logse reestructuraba la formación inicial con un nuevo modelo académico, que igualmente provocó descontento. La nueva estructura académica diseñada resultaba deficiente, pues restringía y dificultaba la preparación inicial. Era "Misión imposible: formar profesionales autónomos y reflexivos en el marco de una diplomatura". (RODRíGUEZ LESTEGÁS, 1998, p. 4). La diplomatura de Maestro/Maestra con siete especialidades era insuficiente para desarrollar el perfil profesional. Añadir que la diplomatura docente estaba en desventaja con la licenciatura establecida para la enseñanza secundaria. De manera que tanto la formación inicial, como la valoración social y las retribuciones marcaban una notable diferencia entre ambos niveles, que denostaba al profesional de la enseñanza primaria. (ibidem, p. 8). Una devaluación de los estudios que desprestigiaba a la profesión y su misión social. Desde las propias instituciones de formación inicial del profesorado se reclamaba la elevación del rango de los estudios, al margen de otros colectivos y expertos, pero sus voces no fueron escuchadas. Los egresados y egresadas de las Escuelas Universitarias de Formación del Profesorado mejoraron su estatus con titulaciones

\footnotetext{
5 Con estrategia y perspectiva para sentar las bases de las competencias a desarrollar resulta clave la modificación de la práctica metodológica: "En realidad, no se trata tanto de enseñar contenidos diferentes, cuanto de utilizar métodos distintos, de orientar la práctica docente hacia esa concepción integrada, aplicada y contextualizada del aprendizaje. No se trata de cambiar los objetivos, sino sobre todo las prácticas de enseñanza y aprendizaje, buscando la interrelación de conocimientos, la movilización de los saberes, la reflexión crítica sobre lo aprendido, la apertura de nuevos caminos hacia el saber. En eso consiste realmente el desarrollo de las competencias básicas". (TIANA, 2011, p. 72).
} 
universitarias (Diplomatura) en un espacio educativo cambiante. La demanda de alargar la formación y equipararla a la de otras titulaciones universitarias, para convertirla en Licenciatura, era un clamor desde las instituciones académicas y algunos sectores profesionales. La diplomatura se queda corta para atender la formación del magisterio. (MOLERO, 2000, p. 79).

La administración educativa no fue capaz de elaborar un plan de formación inicial del magisterio coherente, pues tener conocimiento no equivale a saber transmitirlo. Los diseños de planes de estudio han ignorado la realidad educativa y el proceso de aprendizaje. Los títulos académicos han enmascarado las auténticas problemáticas del ejercicio profesional. Además del perfil y carencia de un modelo "didáctico" formativo óptimo, la modernización de la formación inicial pasó por ampliar la red de especialidades a la vez que restringía el ángulo de actuación docente. Es decir, el abanico de especialidades era mayor mientras se reducía la enseñanza primaria, abarcando seis años de escolaridad, entre los 6 y 12 años (antes de 6 a 14 años). Se trataba de un modelo regresivo. Porque el magisterio volvía a ocupar el espacio académico que había ostentado en décadas anteriores, antes de promulgar la Ley General de Educación (1970). Para atender los nuevos objetivos emanados de la Logse (1990) se recortaron las competencias del magisterio, y la administración educativa persistió en sus reducidas miras académicas. Mientras se reestructuraba todo el sistema educativo en aras del progreso y la evolución experimentada por la sociedad española, la nueva ley no modificó la titulación. (MOLERO, 2000). No se equiparó la titulación al nivel de una Licenciatura. No se propició la homologación universitaria y continuó siendo Diplomatura, pese a que, desde los propios centros, se insistía en su importancia para subsanar la subestimación. Desde las propias instituciones, tanto el profesorado como los directores, reivindicaron el nivel de Licenciatura para los estudios de magisterio. La red de directores de todas las Escuelas universitarias desarrollaron estrategias para conseguir el ansiado objetivo, pero sus esfuerzos resultaron vanos.

En un contexto de cambios y transformaciones sociales era necesaria la acción de los profesionales e instituciones de la educación para continuar en la línea iniciada de la Unión Europea. El Libro Blanco para la Educación y la Formación (1996), de la Comisión Europea, em el Año Europeo de la educación y la formación permanente, se planteaba "¿Cómo preparar a los profesores y formadores ante la evolución de las misiones educativas y la transformación de las herramientas pedagógicas?” (LIBRO BLANCO, 1996, p. 45). No fue hasta comienzos del siglo XXI cuando los estudios de Magisterio se convirtieron en grado. Producto de un nuevo diseño normativo, alcanzaron "la posición de dignidad académica y de prestigio profesional muchas veces deseada y reclamada pero, hasta ahora, nunca conseguida". (MORENO, 2009, p. 43). Así, en el año 2008, adquieren el mismo rango que otros estudios universitarios. Si bien era una reivindicación histórica "la transformación de Magisterio en un título de Grado, de cuatro años, no hizo sino situar a España en condiciones similares a la mayoría de los países desarrollados". (TIANA, 2013, p. 51). La adaptación al Espacio Europeo de Educación Superior tanto en su estructura como en duración de 240 créditos, con lo cual la formación inicial se equipara con los países de la OCDE. (TIANA, 2013). El título tiene carácter generalista y reduce el número de las especialidades a dos (Educación Infantil y Educación Primaria) respecto al plan de 1991, siguiendo la pauta establecida en el Libro Blanco de 2004. No obstante, se podían 
establecer menciones cualificadoras.

La preparación de maestros y maestras es preciso considerarla dentro de la trayectoria política, en consonancia con el proceso de evolución social, y como resultado de la proyección que la sociedad tiene de la educación ${ }^{6}$. Parafraseando a GutiérrezProvecho y López Aguado (2013, p. 32):

Si el objetivo de la formación universitaria es que sus estudiantes adquieran aprendizajes de mayor calidad y se parte del hecho de que estos se consiguen con la utilización de un enfoque profundo, la institución universitaria, empezando por sus docentes, pero no exclusivamente, deben promoverlo. Implica un cambio profundo en las estructuras y en la concepción de los procesos que se producen en las aulas. No es posible modificar la forma en que aprenden los alumnos desde una concepción basada en la enseñanza y en el docente al que el alumno debe ajustarse profundamente. Es necesario modificar sustancialmente el entorno de aprendizaje.

Las políticas educativas han ido construyendo y reconstruyendo el perfil profesional al tiempo que se alteraban las identidades docentes. La formación inicial del profesorado "en la sociedad del siglo XXI adquiere importancia nuclear como eje impulsor de roles activos”. (GONZÁLEZ-PEITEADO; RODRÍGUEZ-LÓPEZ, 2014, p. 71).

El estudiantado tiene variadas razones para acceder a este nivel de estudios, no se trata del marco vocacional en exclusiva. Es una opción dentro del abanico ofertado, sin que exista una implicación personal que ponga de manifiesto la intención de mejora social y política. Las generaciones de estudiantes no siempre se identifican con la carrera profesional. En ocasiones, la elección ha sido motivada por no alcanzar sus objetivos de acceso a otra carrera. Si bien el nivel de exigencias ha aumentado a partir de la Logse y ha superado a otras titulaciones, implica al mismo tiempo una mayor valoración de los estudios, hecho que revaloriza la imagen del magisterio.

La formación inicial de profesorado no siempre se ha desarrollado con el rigor requerido, ni las universidades se han implicado totalmente. Como actividad compleja, no siempre ha sido valorada y reconocida por la sociedad en justa medida. No obstante, la cultura pedagógica de la España democrática ha avanzado, hasta situarse en parámetros homologables con los países europeos. La evolución de las señas de identidad del magisterio, un colectivo que ha incorporado nuevas metodologías y nuevos saberes. El significado de la identidad construida para los profesionales del magisterio, del rol de las maestras y el rol de los maestros, profundiza en el conocimiento de las diferentes modalidades de formación. Se plantea su perfil como guardianes de los valores vigentes, en la defensa de la ideología del sistema a las sucesivas generaciones, así como su contribución en la transmisión de un compromiso político. Sin duda, se ha producido un cambio de mentalidad en sintonía con los avances experimentados en el sistema educativo y la propia sociedad española. (TIANA, 2014).

\footnotetext{
6 "Debido a que la actuación docente debe tener como objetivo prioritario favorecer el aprendizaje, el acto de enseñar debe ser considerado como un encuentro entre una persona que guía en su formación a otra que necesita ayuda para desarrollarse como persona. En consecuencia, la enseñanza deja de ser entendida como una práctica rutinizada para ser concebida como un proceso comunicativo, creativo, reflexivo y complejo". (GONZÁLEZ-PEITEADO; RODRÍGUEZ-LÓPEZ, 2014, p. 71).
} 


\section{Conclusiones}

La formación del magisterio ha ido evolucionando al ritmo de las políticas educativas. Ha significado un cambio en los estilos docentes acentuando la importancia de la formación inicial del magisterio. La continuidad del proyecto de modernización educativa del magisterio que se inició en 1970, a finales de la dictadura, permite considerar el modelo formativo poco avanzado y que paraliza las expectativas de la formación inicial. A ello añadir la escasa preparación del profesorado universitario, responsable de la formación inicial del magisterio. Muchos profesionales se incorporaron a la docencia en las titulaciones de maestros/as especialistas, careciendo de formación e información sobre los profesionales que iban a preparar. El profesorado universitario tenía una preparación condicionada por la cultura externa, tanto a nivel científico como político, regulada por las normas que representaban la modernidad burocrática. La presión de los saberes y de las políticas que promovían las reformas incorporaban las nuevas pedagogías que requería el sistema. La política educativa adoptada para la formación inicial del magisterio con la Ley Orgánica de Ordenación del Sistema Educativo (LOGSE, 1990), una ley promulgada en la etapa de consolidación democrática, generó un nuevo modelo de formación inicial con talante democrático y progresista. Sin embargo, la reforma no mejoró el nivel académico de los egresados ni respondió a las expectativas de los sectores implicados.

La Logse contó con un amplio apoyo político y de algunos sectores profesionales. No obstante, hubo algunos desaciertos. Entre ellos, la formación del profesorado, al no elevar el nivel académico ni diseñar un perfil profesional en sintonía con el contexto social y cultural, tal como reclamaban los sectores implicados. El profesorado de escuelas universitarias de magisterio lamentaba perder la oportunidad de mejorar el rango de los estudios, y desarrollar un currículo más completo y menos fragmentado. La formación inicial del magisterio era un factor fundamental para el desarrollo y modernización del sistema educativo. Además el reconocimiento social a la labor del magisterio requería una mejor preparación. Sin embargo, formar maestros con la diplomatura dificultaba su preparación, porque el espacio académico era insuficiente para construir el modelo pedagógico. El perfil académico, el cambio de estatus, la renovación de las prácticas de enseñanza junto a las nuevas demandas sociales ha influido en las transformaciones de la educación así como en las identidades docentes. El colectivo responsable de la formación de las nuevas generaciones se vio afectado por una normativa que restringía sus competencias educativas, a la vez que no mejoraba la preparación inicial ni la categoría de su rango académico.

La formación inicial del magisterio precisa deconstruir aprendizajes para impulsar nuevos saberes que vinculan la investigación-acción con las competencias profesionales. Parafraseando a Esteve (2007, p. 20), la formación inicial debe centrarse en enseñar a los futuros maestros y maestras a analizar los múltiples factores que influyen en las situaciones de enseñanza, en las que desarrollará su actividad docente. Fijar los objetivos de su identidad profesional para desarrollar su actividad profesional lejos de métodos arcaicos y rutinarios. Había que formar maestros críticos, reflexivos, autónomos y responsables para formar a su vez a las nuevas generaciones de ciudadanos, en el marco de la España plural y democrática que fomente valores éticos y solidarios. En la formación inicial resulta clave transformar el pensamiento pedagógico. Renovar las ideas preconcebidas sobre el magisterio y al mismo tiempo introducir metodologías en la enseñanza universitaria, 
adecuadas con el perfil docente y educativo. Realmente, el verdadero cambio se produciría desde la propia práctica universitaria, a modo de ejemplo a seguir por los futuros maestros y maestras. Al perfil profesional le faltaba coherencia y vínculo con la realidad escolar. Había que conceder mayor importancia a las didácticas y metodologías en las prácticas de enseñanza. Además de la formación pedagógica y psicológica había que potenciar una sólida preparación científica, que domine los saberes propios de su campo, con un nivel intelectual/cultural de acuerdo al rango universitario.

\section{Referencias}

BOLÍVAR, Antonio. La formación inicial del profesorado y las instituciones de Formación. In: ESCUDERO, Juan Manuel; ALBERTO, Luis Gómez (Eds.). La formación del profesorado y la mejora de la educación. Barcelona: Octaedro, 2006. p. 123-154.

La lógica del compromiso del profesorado y la responsabilidad del centro escolar: una nueva mirada. REICE - Revista Electrónica Iberoamericana sobre Calidad, Eficacia y Cambio en Educación, 11(2), p. 60-86, 2013.

COMISIÓN EUROPEA. Libro blanco sobre la educación y la formación. Enseñar y aprender. Hacia la sociedad del conocimiento. Luxemburgo: Oficina de Publicaciones Oficiales de las Comunidades Europeas, 1995.

COROMINAS, Enric; TESOURO, Montserrat; TEIXIDÓ, Joan. Vinculación de los enfoques de aprendizaje con los intereses profesionales y los rasgos de personalidad. Aportaciones a la innovación del proceso de enseñanza y aprendizaje en la educación superior. Revista de Investigación Educativa, 24 (2), p. 443-437, 2006.

ESCUDERO, Juan Manuel (Coord.). Diseño y desarrollo del currículum en la educación secundaria. Barcelona: ICE-Horsori, 1987.

ESTEVE, José Manuel. El choque de los principiantes con la realidad. Cuadernos de Pedagogía, 220, p. 58-63, 1993.

. La formación de profesores: Bases teóricas para el desarrollo de programas de formación inicial. Revista de Educación, n. 350, p. 15-29, 2009.

\section{EDITORIAL. ¿Hasta cuándo? Revista Interuniversitaria de Formación del}

Profesorado, 1, p. 5-7, 1988.

GONZÁLEZ-PEITEADO, Margarita; RODRÍGUEZ-LÓPEZ, Beatriz. La formación inicial de los profesores de lengua extranjera: un espacio para generar estilos de actuación.

Revista Bordón, 66 (4), p. 69-86, 2014.

GUTIÉRREZ-PROVECHO, Lourdes; LÓPEZ AGUADO, Mercedes. La formación inicial de los profesores de lengua extranjera: un espacio para generar estilos de actuación.

Revista Bordón, 66 (4), p. 69-86, 2013.

IMBERNÓN, Francisco. La realidad de la reforma. Instituciones y titulaciones de formación del profesorado. Revista Interuniversitaria de Formación del Profesorado, 29, p. 59-66, 1997.

LEY Orgánica 1/1990, de 3 de octubre, de Ordenación General del Sistema Educativo (Logse). Madrid: Ministerio de Educación y Ciencia.

LIBRO BLANCO. Libro Blanco para la Reforma del Sistema Educativo. Madrid: Centro de Publicaciones, Ministerio de Educación y Ciencia, 1989.

MATERIALES para la Reforma. Educación Infantil. Primaria. Secundaria Obligatoria. Madrid: Centro de Publicaciones, Ministerio de Educación y Ciencia, 1992. 
MARAVALL, José Ma. La reforma de la enseñanza. Barcelona: Laia, 1984.

MOLERO PINTADO, Antonio. La formación del maestro español, un debate histórico permanente. Revista de Educación, n. extra (1), p. 59-82, 2000.

MORENO, Amparo. La formación del profesorado: una respuesta adecuada a los nuevos tiempos. In: PUELLES BENÍTEZ, Manuel de (Coord.). Profesión y vocación docente. Presente y futuro. Madrid: Biblioteca Nueva, 2009. p. 43-63.

OECD. Creating Effective Teaching and Learning Environments. First Results from Talis. Paris: OECD, 2009.

PALOMERO PESCADOR, José Emilio. Editorial. Revista Interuniversitaria de Formación del Profesorado, 2, p. 7-11, 1988.

PAPELES para el Debate. 4 vols. Madrid: Ministerio de Educación y Ciencia, 1987.

PLAN de Investigación Educativa y de Formación del Profesorado. Madrid: Ministerio de Educación y Ciencia, 1987.

PROYECTO de Reforma del Sistema Educativo. Propuesta para Debate. Madrid: Ministerio de Educación y Ciencia, 1987.

RODRÍGUEZ LESTEGÁS, Francisco. La formación inicial de los maestros en la actualidad: historia de una inconsecuencia. Revista Electrónica Interuniversitaria de Formación del Profesorado, p. 1-9, 1998. Disponible en:

<http://www.aufop.com/aufop/uploaded_files/articulos/1224540880.pdf>. Acceso en: 20 dez. 2016.

RODRÍGUEZ MARCOS, Ana; SANZ LOBO, Estefanía; SOTOMAYOR SÁEZ, Mํㅡ. Victoria (Coords.). La Formación de los Maestros en los Países de la Unión Europea. Madrid: Narcea, 1998.

TIANA FERRER, Alejandro. Análisis de las competencias básicas como núcleo curricular en la educación obligatoria española. Revista Bordón, 63 (1), p. 63-75, 2011. 23, 2014 .

Leyes y reformas: de la LGE a la LOMCE. Cuadernos de Pedagogía, 451, p. 20 -

. Los cambios recientes en la formación inicial del profesorado en España: Una Reforma incompleta. Revista Española de Educación Comparada, 22, 39-58, 2013.

TORRES, Jurjo. La justicia curricular. El caballo de Troya de la cultura escolar. Madrid: Morata, 2011.

ZABALZA, Migual Angel. El practicum en la Formación de los Maestros. In: MARCOS, Ana Rodríguez; LOBO, Estefanía Sanz; SOTOMAYOR SÁEZ, Mํㅡㄴ Victoria (Coords.). La Formación de los Maestros en los Países de la Unión Europea. Madrid: Narcea, 1998. p. 169-202.

ZABALZA, Miguel Angel. Diseño y desarrollo curricular. Madrid: Narcea, 1999. 


\section{ANEXO}

\section{Planes de Estudios de Magisterio Logse.}

Real Decreto 1440/1991, de 30 de agosto (BOE, 11 de octubre).

\section{Título de Maestro-Especialidad de Educación Infantil}

MATERIAS TRONCALES COMUNES DEL TITULO DE MAESTRO EN TODAS SUS ESPECIALIDADES.

1. Bases psicopedagógicas de la Educación Especial

2. Didáctica General

3. Organización del Centro Escolar

4. Psicología de la Educación y el desarrollo en edad escolar

5. Sociología de la Educación

6. Teoría e instituciones contemporáneas de educación

7. Nuevas tecnologías aplicadas a la educación

8. Prácticum

\section{MATERIAS TRONCALES DE LA ESPECIALIDAD}

1. Conocimiento del medio natural, social y cultural

2. Desarrollo de la Expresión Musical y su didáctica

3. Desarrollo de la Expresión Plástica y su didáctica

4. Desarrollo de las Habilidades Lingüísticas y su didáctica

5. Desarrollo del Pensamiento Matemático y su didáctica

6. Desarrollo psicomotor

7. Literatura Infantil

8. Prácticum

\section{Título de Maestro-Especialidad de Educación Primaria}

\section{MATERIAS TRONCALES DE LA ESPECIALIDAD}

1. Ciencias de la Naturaleza y su didáctica

2. Ciencias Sociales y su didáctica

3. Educación Artística y su didáctica

4. Educación Física y su didáctica

5. Idioma Extranjero y si didáctica

6. Lengua y Literatura y su didáctica

7. Matemáticas y su didáctica

8. Prácticum 


\section{Título de Maestro-Especialidad de Lengua Extranjera}

MATERIAS TRONCALES DE LA ESPECIALIDAD

1. Conocimiento del medio natural, social y cultural

2. Educación Artística y su didáctica

3. Educación Física y su didáctica

4. Idioma Extranjero y si didáctica

5. Lengua y Literatura y su didáctica

6. Matemáticas y su didáctica

7. Fonética (idioma extranjero correspondiente)

8. Lingüística

9. Morfosintáxis y Semántica

10. Prácticum

\section{Título de Maestro-Especialidad de Educación Física}

MATERIAS TRONCALES DE LA ESPECIALIDAD

1. Conocimiento del medio natural, social y cultural

2. Educación Artística y su didáctica

3. Educación Física y su didáctica

4. Idioma Extranjero y si didáctica

5. Lengua y Literatura y su didáctica

6. Matemáticas y su didáctica

7. Aprendizaje Motor

8. Bases Biológicas y fisiológicas del movimiento

9. Educación Física y su práctica

10. Teoría y Práctica del acondicionamiento físico

11. Prácticum

\section{Título de Maestro-Especialidad de Educación Musical}

MATERIAS TRONCALES DE LA ESPECIALIDAD

1. Conocimiento del medio natural, social y cultural

2. Educación Artística y su didáctica

3. Educación Física y su didáctica

4. Idioma Extranjero y si didáctica

5. Lengua y Literatura y su didáctica

6. Matemáticas y su didáctica

7. Agrupaciones Musicales

8. Formación Rítmica y Danza

9. Formación Vocal y Auditiva

10. Historia de la Música y del Folklore 
11. Lenguaje Musical

12. Prácticum

\section{Título de Maestro-Especialidad de Educación Especial}

\section{MATERIAS TRONCALES DE LA ESPECIALIDAD}

1. Aspectos Didácticos y Organizativos de la Educación Especial

2. Aspectos Evolutivos y Educativos de la Deficiencia Auditiva

3. Aspectos Evolutivos y Educativos de la Deficiencia Mental

4. Aspectos Evolutivos y Educativos de la Deficiencia Motórica

5. Aspectos Evolutivos y Educativos de la Deficiencia Visual

6. Educación Física en alumnos con necesidades educativas especiales

7. Expresión Plástica y Musical

8. Trastornos de Conducta y Personalidad

9. Tratamientos Educativos de los Trastornos de la Lengua escrita

10. Prácticum

\section{Título de Maestro-Especialidad de Audición y Lenguaje}

\section{MATERIAS TRONCALES DE LA ESPECIALIDAD}

1. Anatomía, Fisiología y Neurología del Lenguaje

2. Aspectos Evolutivos del Pensamiento y el Lenguaje

3. Desarrollo de Habilidades Lingüísticas

4. Lingüística

5. Psicopatología de la Audición y el Lenguaje

6. Sistemas Alternativos de Educación

7. Tratamiento Educativo de los trastornos de la Audición y del Lenguaje

8. Tratamiento Educativo de los trastornos de la Lengua oral y escrita

9. Prácticum

TERESA GONZÁLEZ PÉREZ es profesora Titular acreditada Catedrática de Universidad, Área de Teoría e Historia de la Educación, Departamento de Historia y Filosofía de la Ciencia, la Educación y el Lenguaje (Universidad de La Laguna-España). Sus líneas de investigación comprenden Política Educativa, Historia de la Educación (formación del magisterio), Estudios sobre las mujeres y Estudios Culturales. Autora de diversos libros y de numerosos artículos publicados en revistas nacionales e internacionales (Educación, Bordón, RIFP, History of Education \& Children's Literature, História da Educação). Participa regularmente en encuentros cientificos en Universidades Europeas y Americanas.

Endereço: Facultad de Educación, Universidad de La Laguna, Campus Central - Avda. de la Trinidad, s/n, 38204, San Cristóbal de La Laguna - Tenerife - España.

E-mail: teregonz@ull.edu.es 
Recebido em 27 de janeiro de 2017.

Aceito em 09 de junho de 2017.

(c) (i) 